\title{
Chine - Brésil: je t’aime, moi non plus.
}

\section{China - Brasil: te amo, mas nem tanto.}

Pierre Salama - Doctor en Economie, Professeur Emérite des Universités, Université de Paris 13, Paris, France. Publications surtout sur l'état, l' économie et le dévélopment. Latino-américaniste. E-mail: pierresalama@gmail.com

\section{Resumo}

Le succès économique de la Chine faitil le malheur économique du Brésil, ou bien faut-il chercher ailleurs les causes de la détresse dans laquelle s'enfonce aujourd'hui le Brésil? fait penser à la «maladie hollandaise». La voie pour sortir du piège rentier est étroite mais elle existe. Les gouvernements brésiliens ont préféré ne pas l'emprunter, persuadés que la hausse du cours des matières premières allait se poursuivre et qu'elle permettrait, sans faire des réformes structurelles de mener une politique visant à réduire les inégalités salariales, de diminuer la pauvreté en laissant les $1 \%$ les plus riches s'enrichir davantage, sans se préoccuper de la perte compétitivité. Les relations asymétriques, croissantes, avec la Chine ont permis la crise mais ne l'ont pas créée.

\section{Palavras-chave}

Brasil. China. Desenvolvimento. Estado. Economia global.

\section{Resumo}

¿Éxito económico de China hizo la difícil situación económica del Brasil, o hay buscar en otro lado las causas de la dificultad que ahora se hunde el Brasil? es una reminiscencia de la 'enfermedad holandesa'. La manera de salir de la trampa rentista es estrecha pero existe. Los gobiernos brasileños han preferido no escoger una via que favorece la industrialización, convencidos de que la subida de las materias primas continuarían, sin hacer las reformas estructurales para aplicar una política destinada a reducir la desigualdad de los salarios, reducir la pobreza y dejando el $1 \%$ rico enriquecería más, sin preocuparse por la pérdida de competitividad. Las relaciones asymetricas, crecientes con la China han permitido la crisis de hoy sino que la crearon.

\section{Keywords}

Brasil. China. Desarrollo. Estado. Economia global. 


\section{INTRODUCTION}

La Chine et le Brésil, deux pays aux parcours économiques différents qui entretiennent des relations étroites mais asymétriques entre eux, et dont l'un poursuit un parcours économique où les restructurations sont l'alpha et l'oméga de sa croissance, et l'autre, profitant des demandes que lui adresse la Chine, parait régresser vers le passé en se re-spécialisant sur des activités de rente. Le succès (économique) de l'un fait-il le malheur (économique) de l'autre, ou bien faut-il chercher ailleurs les causes de la détresse dans laquelle s'enfonce aujourd'hui le Brésil?

\section{DE QUELQUES FAITS STYLISÉS: CHINE BRÉSIL, CE QUI LES DIFFÉRENTIE}

1. Le PIB par tête: dans les années 1980, l'Amérique latine est rattrapée puis dépassée par les dragons asiatiques (Corée du sud, Taïwan, Hong-Kong et Singapour). Cependant, dans les années 1990-2000, l'Amérique latine renoue avec une croissance modérée et le revenu par habitant des principaux pays (Argentine, Brésil, Mexique, Colombie...) s'établit en 2012 à un peu plus du quart du revenu par tête des Etats-Unis alors que ceux de la Corée du sud et de Taïwan se situent autour de $70 \%$ pour le premier et $80 \%$ pour le second de celui des Etats-Unis. Malgré cette croissance plus élevée, l'Amérique latine se marginalise. En 1980, le revenu par tête de la Chine s'élève à 306,7 dollars, mesuré en parité de pouvoir d'achat (PPA) et celui du Brésil à 4809,6 dollars PPA. En 2015, il est très proche de celui du Brésil 14107,4 dollars PPA et15614 dollars PPA respectivement (FMI). Entre 1980 et 2015 le PIB par tête a été multiplié par 46 en Chine, il ne l'a été que par 3,25 au Brésil.

Les parcours économique sont très différents. En Chine, la croissance est portée chez l'un par un essor impressionnant des exportations de produits manufacturés et un surinvestissement ; au Brésil, dans les années 2000, elle l'est par les exportations des matières premières et freinée par les investissements insuffisants. Croissance très élevée chez l'un, croissance globalement molle chez l'autre, bien que légèrement supérieure à celle des années 1990.

Dès l'arrivée du Président Lula da Silva au pouvoir (2003), la croissance s'accélère légèrement, la pauvreté baisse drastiquement mais contrairement à ce qui se dit officiellement, les riches deviennent plus riches et la part des 1\% dans le revenu des plus riches s'accroit comme dans l'ensemble des pays avancés, le Brésil ne faisant pas exception sauf dans sa capacité à diminuer la pauvreté. Nombre d'économistes s'interrogent alors sur la possibilité que le Brésil devienne un 
nouvel eldorado. Le mythe s'effondre dès 2011 et les rêves s'envolent, le miracle devient mirage, voire cauchemar dès 2014.

2. La démographie: la Chine (1,4 milliard d'habitants) est très peuplée comparée au Brésil (207 millions). Lorsqu'on tient compte des inégalités de revenus et de leurs évolutions, les conséquences de la démographie sur la dimension des marchés intérieurs sont différentes dans les deux pays:

a. Les inégalités de revenus en Chine se rapprochent de celles observées au Brésil. De ce point de vue la Chine s'est latino-américanisée, la croissance produisant des inégalités de plus en plus importantes. Celles- ci cependant ne constituent pas un obstacle à la poursuite de cette croissance précisément en raison de l'importance des demandes de biens de consommation, durable ou non, de biens intermédiaires et de biens d'équipement liée à celle de la population. Les capacités de production oisives en forte augmentation sont dues à un surinvestissement consécutif au plan de relance massif de 2008-2009, mais aussi à des politiques de localisation décidées au niveau provincial aboutissant à une multiplication des usines produisant le même produit dans chacune des provinces de Chine qu'à une insuffisance de la demande et à la spéculation immobilière. Nonobstant ce problème important, l'essor vif de la production industrielle vérifie la loi de Kaldor: plus d'industrie, davantage de productivité et d'économies d'échelle, plus de croissance. Bien que le Brésil ait également une population importante (207 millions d'habitants), comparée à celle des autres pays latino-américains, cette loi est moins vérifiée. Les inégalités de revenus sont très importantes et la dimension du marché intérieur peut être insuffisante, relativement à l'offre nécessaire pour bénéficier des économies d'échelle, pour la fabrication de certains produits sauf à compléter cette dimension de la demande interne par celle des marchés extérieurs comme cela s'est fait en Corée du sud par exemple. La concurrence internationale, stimulée par la libéralisation commerciale, l'appréciation de la monnaie, l'allégement des subventions, rend encore plus difficile la production interne dans des conditions de rentabilité suffisante, l'augmentation des demandes des différentes catégories sociales, celle des produits intermédiaires et de biens d'équipement étant alors de plus en plus satisfaite par un essor des importations rendue possible par la disparition de la contrainte externe, consécutive à l'augmentation des exportations de produits primaires et à "l'aisance" en devises due à la montée des investissements dans le pays. La croissance dans les années deux mille est alimentée par l'essor des activités de rente, la désindustrialisation s'affirmant et la productivité évoluant très modestement. 
b. Le taux de croissance démographique, selon l'Insee, en 2011 est de l'ordre 0,48 en Chine malgré la politique de l'enfant unique, remise en question aujourd'hui, il est de 1,1 au Brésil. Les taux de fécondité dont différents. En Chine, la politique de l'enfant unique décidée en 1979 pour enrayer l'explosion démographique, a conduit à un vieillissement de la population et à une transformation de la pyramide des âges devenant progressivement un obstacle à la poursuite de la croissance, le rapport actifs/inactifs devient de plus en plus faible, d'où l'abandon de cette politique en 2015. Le Brésil connait une transition démographique assez rapide: selon les données de l'IBGE, le taux de fécondité était en 1980 de 4,10, en 1990, de 2,80, en 2000 de 2,40 et en 2015 de 1,70 soit un taux équivalent à celui observé en Europe. Mais comme la transition a été rapide, la population en âge de travailler (entre 15 et 64 ans) est très importante en terme relatif, soit 140,9 millions sur une population de 205 millions). Le Brésil doit donc offrir des emplois en nombre à cette population, ce qui implique un taux de croissance important d'autant plus que le pays a un taux d'urbanisation plus élevé que celui des pays industrialisés. Pour un taux de croissance faible, le risque d'une montée du chômage est élevé même avec un taux de croissance de la productivité faible. La crise, initiée en 2014, se traduit en 2015-2016 par un doublement du taux de chômage affectant plus particulièrement les jeunes et les jeunes diplômés. A l'inverse, en Chine, une fois ralentie la migration des campagnes vers les villes, le manque de main d'œuvre se fait sentir dans certaines provinces, à l'exception des provinces côtières où le ralentissement économique est important.

3. Les richesses naturelles sont très différentes dans les deux pays. Mises à part les "terres rares", le charbon, la Chine ne possède pas de matières premières à la hauteur de ses besoins. Aussi est elle contrainte de les importer pour alimenter sa croissance et pour satisfaire les nouveaux besoins liés aux changements de modes de consommation eux-mêmes conséquences de l'essor du revenu par tête. A l'inverse, le Brésil est très riche en matières premières, que celles-ci soient d'origine minérale ou agricoles. C'est ce qui explique à la fois l'essor de la production de matières premières en vue de l'exportation, un retour, à l'égal de la spécialisation internationale du $\mathrm{XIX}^{\circ}$ siècel, vers une primarisation de l'économie, cette fois utilisant des techniques ultra sophistiquées, qu'on a pu désigner par le terme de "commodities consensus" (M.Svampa') et enfin des investissements directs de la Chine au Brésil afin de d'assurer l'approvisionnement en matières premières, que ce soit par l'achat de terres ou bien de mines. La Chine est ainsi

Svampa M.(2013): «Consenso de los commodities y lenguajes de valoracion en America latina », Nueva Sociedad, ${ }^{\circ} 244$ 
devenue le premier partenaire commercial du Brésil, 19\% de ses exportations lui sont destinées en 2013, devançant les Etats-Unis (10\%). C'est le cas également du Chili (25\%), de l'Uruguay (22\%) et du Pérou (18\%, à égalité avec les Etats-Unis). Ce n'est pas encore le cas de la Colombie, les Etats Unis demeurant le premier partenaire $(32 \%)$ suivie de la Chine (9\%), source FMI - DOTS. Il en résulte une vulnérabilité plus ou moins forte selon à la fois le degré d'ouverture et la part des exportations vers la Chine sur les exportations totales. Comme on peut le voir, elle est élevée pour le Brésil (BR), mais moins qu'au Chili (CL) où le degré d'ouverture plus élevé et la part des exportations plus importante, ou encore au Venezuela (VE), au Pérou (PE) et en Uruguay (UY).

\section{DES RELATIONS ÉCONOMIQUES DE PLUS EN PLUS ÉTROITES MARQUÉES PAR UNE DOUBLE ASYMÉTRIE}

La Chine est donc devenue le premier client du Brésil. Les relations qu'entretiennent les deux pays sont de plus en plus étroites dans les années 2000. Les exportations brésiliennes a destination de la Chine étaient inférieurs à 1 milliard de dollars en 2000, elles dépassent 40 milliards de dollars en 2013. La Chine était le $12^{\circ}$ partenaire du Brésil pour les exportations et le $11^{\circ}$ pour les importations en 2000, elle est son premier partenaire dès 2009 pour ses exportations et le premier en 2012 pour ses importations. Ses exportations vers la Chine correspondent à 18 ù de ses exportations globales et ses importations de Chine à 16\% de ses importations totales en 2014 (Ocde/ Caf/Cepal, 2016). Le solde commercial entre les deux pays de négatif en 2000 (137 millions de dollars) est largement positif, sauf en 2008, à mesure que les ventes de matières premières à la Chine progressent. Il s'établit à 11,(2 milliards de dollars en 2011 et déclinent fortement ensuite, la valeur de ses exportations chutant vers la Chine et ses importations régressant moins que ses exportations pour se fixer à 3,27 milliards de dollars en 2014 et 4,88 milliards de dollars en 2015 (CEPII).

Les relations commerciales entre la Chine et le Brésil se caractérisent par une double asymétrie : la chine vend des produits manufacturés au Brésil et lui achète des matières premières. Si la Chine est le partenaire le plus important du Brésil, ce n'est pas le cas du Brésil. Ces deux asymétries ne sont pas sans conséquences sur les négociations entre les deux pays.

La structure des exportations brésiliennes a destination de la Chine est dominée par la vente des matières premières et des produits intensifs en ressources naturelles, soit respectivement $66,7 \%$ et $13,8 \%$ en 2000 et $83,7 \%$ et 
10,3\% en 2011. A l'inverse, la structure des exportations de la Chine vers le Brésil est dominée par la vente de produits manufacturés, les ventes de produits primaires et de biens intensifs en ressources naturelles ne représentant que $8 \%$ et $13,6 \%$ respectivement des importations en provenance de Chine en 2000 et $2,5 \%$ et $9,6 \%$ respectivement en 2011 , alors que celles de produits manufacturés intensifs en recherche développement représentaient 20,4\% (Secex/MDIC).

Le Brésil fournit à la Chine 75\% de ses importations de produits agricoles en provenance d'Amérique latine, l'Argentine 16\% en 2013. Les exportations de soja vers la Chine correspondent à $37 \%$ de ses exportations vers ce pays. Les exportations de produits agricoles sont fortement concentrées sur le soja: $78 \%$ pour les graines de soja et tourteaux et $6 \%$ pour l'huile de soja en 2011 (Ocde/Caf/Cepal, 2014 et 2016).La vente des graines de soja s'élevait à 15\% de l'ensemble des exportations du Brésil au $1^{\circ}$ septembre $2014^{2}$. Les ventes ont particulièrement augmenté entre 2002 et 2011 passant de 896 millions de dollars à 9513 millions de dollars pour les premiers et de 127 millions de dollars à 660 pour l'huile de soja. Les exportations agricoles étant très concentrées, Brésil est particulièrement vulnérable à l'évolution du cours de cette matière première. Bien que le volume des ventes latino-américaines de soja se soit accru de 28\% en 2015, la valeur de celles-ci a baissé de 7\% (in China-Latin American Economic Bulletin, 2016-3 à partir des données de UN-Comtrade data), l'augmentation importante des volumes ne compensant pas la chute des cours. Entre 2013 et 2015, le cours de la graine de soja est passé de 538 dollars la tonne métrique à 391 dollars.

Le Brésil exporte également d'autres matières premières parmi lesquelles le minerai de fer reste le plus important. La valeur des exportations de minerai de fer et dérivés s'élevait à 35\% de ses exportations vers la Chine en 2014. La chute du cours des minerais de fer a été impressionnante. Avec un indice 100 en 2010, son cours atteint 28 en décembre 2015 (voir Commodity Market Outlook, op.cit), et ce n'est que depuis fin 2015 que la courbe semble légèrement s'inverser. Bien que le volume de minerai de fer ait augmenté de 10\% entre 2014 et 2015, la chute en valeur des exportations a été particulièrement élevée : - 34\% (in ChinaLatin American...op.cit) ${ }^{3}$.

\footnotetext{
2 La production de graines de soja s'est très rapidement accrue au Brésil : en 1980/1981 la production s'élevait à 15,2 millions de tonnes métriques, en 2014/2015 à 100 millions de tonnes métriques dont la moitié était exportée, principalement vers la Chine qui, à cette date, importait 78,4 millions de tonnes métriques (voir Commodity Market Outlook, Quaterly Report, World Bank, Jan.2016).

3 Les perspectives d'évolution sont assez pessimistes. Certes la consommation de minerai de fer de la Chine est considérable : $43,9 \%$ de la consommation mondiale en moyenne entre 2010 et 2014 - elle était seulement de 6,4\% en moyenne entre 1990 et 1994 - mais elle décline fortement depuis quelques années en raison de la surproduction dans le secteur sidérurgique.
} 
Cours de la graine de soja en dollars par tonne métrique, 2005-2015

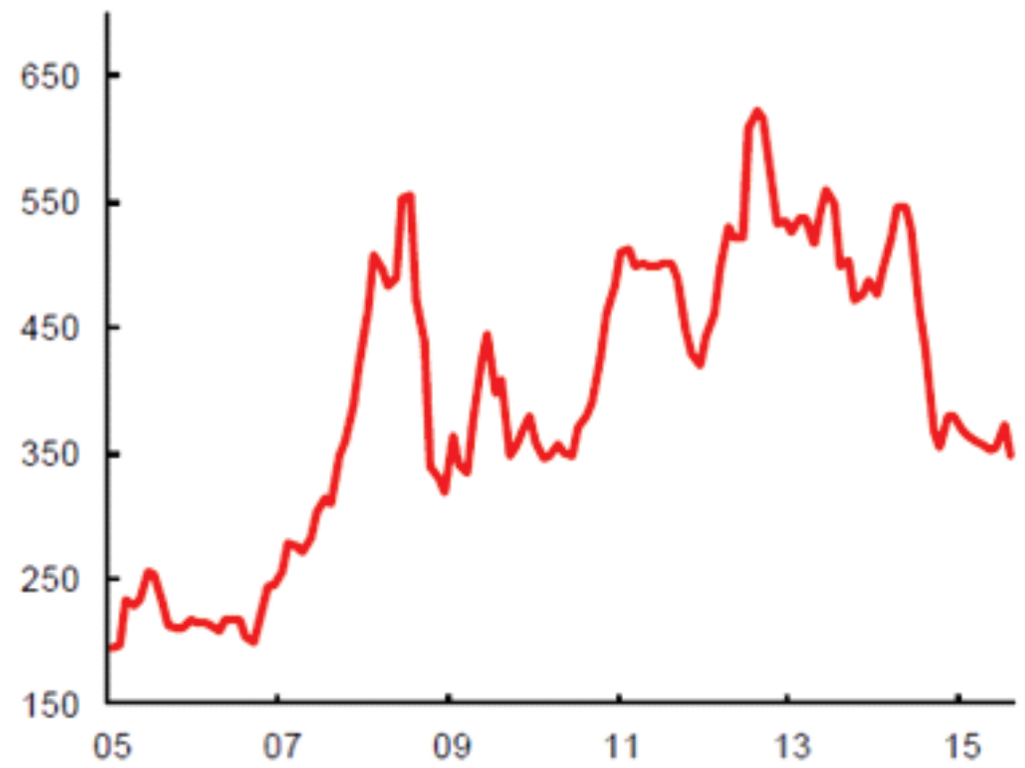

Source: Commodity Market Monthly, IMF, sept 2015

Cours du minerai de fer en dollar par tonne métrique, 2005-2015

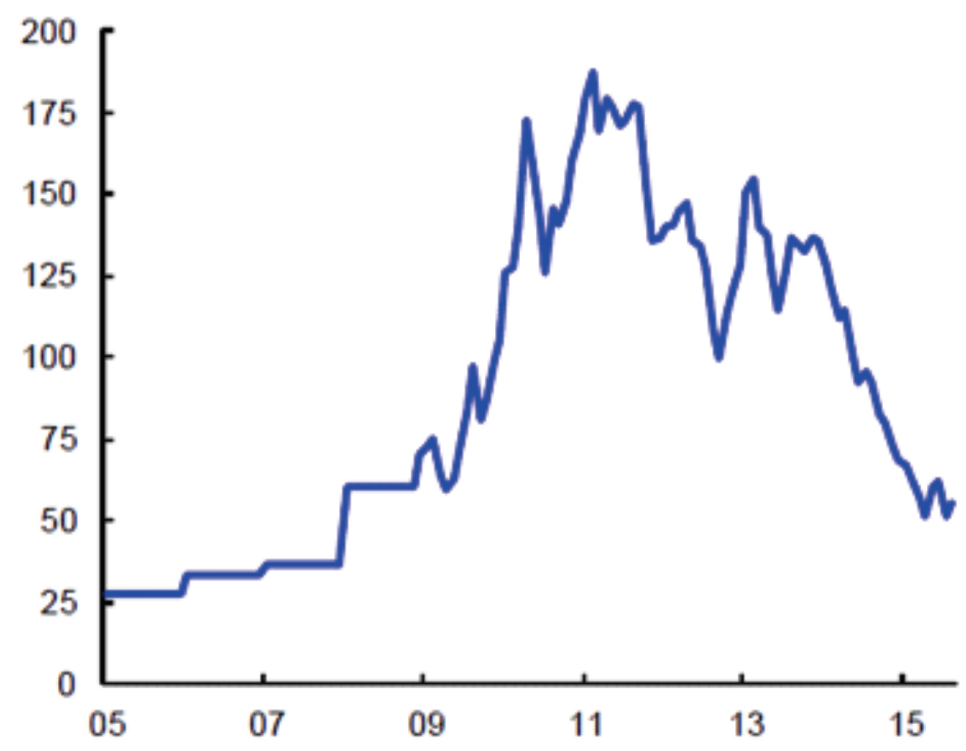

Source: Commodity Market Monthly, IMF, sept 2015 
Comme on peut le voir dans le graphique ci-dessous, la vulnérabilité du Brésil vis-à-vis de la Chine s'explique (pour partie) par l'importance de son commerce avec la Chine. Le Brésil est apparemment moins vulnérable que le Pérou, le Chili, l'Uruguay dans la mesure où son degré d'ouverture est moins élevé que dans ces pays, les effets de contagion étant dès lors moins importants.

Cependant, il faut se garder d'une approche qui se limiterait à l'analyse de ces deux seules variables. Au-delà du degré d'ouverture et de l'importance relative du commerce avec la Chine, il faut considérer les effets du commerce avec la Chine sur le tissu industriel, la nature des produits exportés, comment ils participent à la chaine internationale de la valeur. Le Mexique par exemple exporte très peu à la Chine faute de matières premières, par contre il importe énormément d'elle des produits de l'industrie de transformation. Pour un dollar qu'elle exporte à la Chine, elle importe l'équivalent de 10 dollars d'elle. Les relations commerciales qu'elle entretient avec la Chine, plus exactement la manière dont se pratique son commerce, participent à sa désindustrialisation de nombre de secteurs industriels tournés vers son marché intérieur. Dès lors, son secteur industriel affaibli est source de vulnérabilité. Comme on peut le voir dans le graphique suivant, le Mexique est plus menacé que le Brésil, son indice de "menace concurrentielle" étant plus élevé.

Evolution de l'indice de "menace concurrentielle" en \%, 2000, 2005, 2010, 2013 Mexique et Brésil

\begin{tabular}{l|c|c|c|c}
\hline & 2000 & 2005 & 2010 & 2013 \\
\hline MEXIQUE & 47.59 & 44.812 & 46.168 & 41.942 \\
\hline BRESIL & 26.174 & 21.76 & 12.726 & 14.197 \\
\hline
\end{tabular}

Source: Ocde/Caf/Cepal, 2016, p.110. Cet indice se réfère aux travaux de Lall, repris par Gallagher, se calcule comme le pourcentage des secteurs (4 digits) dans lesquels la Chine a un taux de croissance supérieur à la croissance des exportations de tel ou tel pays dans la période $2000-2012$

Le Brésil, ainsi que l'ensemble des pays latino-américains, participe moins à la chaine internationale de valeur que la Chine. On distingue deux type de participation à cette chaine : celle par l'arrière et celle par l'avant. La première cherche à mesurer les biens importés qui sont incorporés dans les exportations d'un pays en pourcentage de ses exportations brutes, la Chine ou le Brésil, ici. La seconde, elle, cherche à mesurer les biens exportés par un pays incorporés dans les exportations de ce dernier en pourcentage de ses exportations brutes. La 
participation par l'arrière est de 11,4\% en 2000 et 10,7\% en 2011 au Brésil alors qu'elle est de $37,2 \%$ et de $32,1 \%$ à ces dates en Chine. La participation un peu plus faible en 2011 qu'en 2000 est révélatrice de l'effort de la Chine pour intégrer davantage ses lignes de production.

La participation à la chaine de valeur par l'avant est pour le Brésil plus importante que celle de la Chine : 17,1\% en 2000 et 24,5\% en 2011 au Brésil contre 10,8\% et 15,6\% pour la Chine à ces deux dates (Ocde, Caf, Cepal, op cit, chapitre 4). Avec la reprimarisation de son économie, le Brésil exporte en effet de plus en plus des biens, en l'occurrence ici des matières premières, vers d'autres pays, dont la Chine, qui les incorporent pour la production de leurs exportations. De ces données, on déduit à la fois que la fragmentation est plus faible au Brésil qu'en Chine, et que portant moins sur des produits à forte élasticité demande par rapport au revenu, elle est moins sujette à une insertion dynamique dans la division internationale du travail que la Chine et la plupart des économies asiatiques. Allant plus loin, sa complémentarité avec la Chine n'est pas de l'ordre du «gagnant-gagnant». L'une perd à la fois parce que sa spécialisation porte sur des produits de rente et qu'à terme plus ou moins long, les retournements de prix ont lieu, et parce que ce faisant, elle rend possible un affaiblissement de son appareil industriel. L'autre gagne parce que se nourrissant de matières premières qui lui font défaut, elle joue sur la flexibilité de son appareil industriel, sur sa volonté d'intégration, pour abandonner la production de biens nécessitant peu de capital et beaucoup de main d'œuvre non qualifiée vers la production de produits plus sophistiqués davantage demandés, à l'égal de ce que firent en leur temps la Corée du sud ou bien Taïwan.

L'insuffisance de matières premières, hors le charbon et les terres rares, vulnérabilisent la Chine. Aussi n'est-il pas étonnant que depuis quelques années elle utilise une partie de ses réserves internationales pour multiplier les investissements directs dans les pays riches en matières premières, favoriser le développement des infrastructures portuaires, de chemin de fer, qu'elle investisse dans l'achat de terres, afin de sécuriser son environnement.

Il est très difficile d'avoir une idée exacte del'importance des investissements étrangers directs de la Chine en Amérique latine. Si on s'en tient aux données officielles, l'Amérique latine n'est pas une destination majeure : 15,3\% des IED chinois réalisés contre 65\% pour les pays asiatiques en 2010, le Brésil étant marginal ( $0,7 \%$ des IED chinois, source Mofcon of China). Ces données sont cependant sous-évaluées : une très grande partie des investissements passe par Hong Kong, le Luxembourg, les îles Caïman, les îles Vierges et ne sont pas nécessairement comptabilisées par le Mofcon of China, ainsi en est-il de l'achat de 40\% de 
Repsol-Brésil par la Chine qui apparait comme un achat ... luxembourgeois. Plus globalement, les données de l'ONECH (2014) révèlent qu'entre 2010 et 2013 les Iles Vierges reçoivent $51 \%$ des investissements étrangers directs chinois destinés à l'Amérique latine, les Iles Caïman 32\%, le Brésil n'en recevant que 3\%. Si, comme la Cepal l'a fait en 2015, on «redresse» ces données, on observe qu'entre 2010 et 2015, le Brésil a en réalité reçu 56\% des investissements étrangers chinois, le Pérou 16\%, l'Argentine 15\%, le Venezuela et la Colombie chacun 5\% ${ }^{4}$

Depuis 2010 ces investissements, ainsi que les prêts, ont connu un certain essor sans qu'on puisse avec précision évaluer leur importance. Les promesses de prêts se sont accrues, leurs réalisations également sans qu'on connaisse exactement l'échelonnement du passage des unes aux autres. Ces prêts sont parfois remboursés en matières premières comme c'est le cas du Venezuela. Ils se sont accrus de 2013 à 2014 de $71 \%$ en 2014 atteignant 22 milliards de dollars, dont 8,6 destinés au Brésil, 7 à l'Argentine et 5,7 au Venezuela selon China Latin -America Finance Database report. Les investissements directs se sont accrus pour deux rasions : la dépréciation des monnaies latino-américaines rend moins coûteux l'investissement, les négociations entre la Chine et les Etats latinoaméricains, notamment le Brésil, aboutissant à des promesses d'investissements directs, notamment dans le secteur industriel, pour compenser la montée des importations de Chine.

Selon les calculs effectués par le Financial Times et le Bureau Van Dijk (voir China-Latin American bulletin, op.cit), les investissements étrangers directs chinois, de plus de 10 millions de dollars, à destination de l'ensemble de l'Amérique latine, de type Greenfield seraient de 31,3 milliards de dollars et les fusionsabsorptions de 18,8 milliards de dollars, entre 2011 et 2015. La composition de ces deux types d'investissement n'est pas la même. Les investissements de type Greenfield concernent principalement le secteur manufacturier (20\%), l'agriculture $(18 \%)$, la logistique (15\%), les communications (14\%), la finance et l'immobilier (14\%), les acquisitions privilégient le pétrole (39\%), le secteur extractif (37\%), ces deux premières destinations représentant plus des trois quarts de l'ensemble des acquisitions, le secteur manufacturier ne représentant que 1\%. On voit que la stratégie de la Chine est différente de celle développée vis-à-vis des pays avancés. L'achat d'entreprises étrangères n'a pas pour objectif l'acquisition de technologies étrangères mais de s'assurer principalement un approvisionnement en matières premières. Dans ces deux secteurs les fusions absorption sont proportionnellement beaucoup plus importantes que celles opérée par le reste

$4 \quad$ Voir E. Dussel Peters et S. Ortiz Velasquez (coordinateurs) : Monitor de la OFDI de China en Mexico, n , Mars 2016. 
du monde, celles-ci s'élevant à 15\%. A l'inverse, les fusions-absorptions dans le secteur manufacturier, quasi - inexistantes en provenance de Chine, s'élèvent à 13\% pour le reste du monde. Les investissements directs chinois destinés au secteur manufacturier se dirigent principalement vers le Brésil et dans une moindre mesure l'Argentine.

III. La Chine responsable de la faible croissance du Brésil et de sa désindustrialisation?

Il n'est nul besoin de se référer aux étapes de développement théorisées en son temps par Rostow pour reconnaitre qu'il y a des parcours porteurs d'avenir et d'autres qui le sont moins ou pas du tout. On peut regrouper les économies en quatre catégories : économies attardées (émergentes ou non), tigres, champions et enfin économies mûres à partir d'un graphique en quatre quadrants, en ordonnée, la densité industrielle, mesurée en valeur ajoutée par habitant en dollars et, en abscisse, le poids du secteur manufacturier par rapport au PIB, le croisement de l'ordonnée et de l'abscisse se situant à 3000 dollars et 18\%. Les principales économies émergentes se situent dans le premier quadrant, proche du second. Leur densité industrielle n'est pas très importante et le poids de leur manufacture dans le PIB est également faible, ce n'est pas le cas des tigres dont la densité est certes encore relativement faible mais dont le poids de leur manufacture est plus important. Leurs succès, en termes de croissance, de faible volatilité et de durabilité de celle ci suggèrent d'accroitre l'importance relative de l'industrie. L'étape supérieure est la conséquence logique de cette croissance plus élevée mais déjà se manifestent les effets d'une désindustrialisation relative, le poids des services devenant de plus en plus important. L'étape finale, celle d'économies mûres, se caractérisant par une tendance à une stagnation séculaire. Elle concerne surtout les économies avancées.

Ce parcours économique possible - de quadrant à quadrant - que suggère ce graphique est une application de la loi de Kaldor que nous avons évoquée précédemment. Les économies latino-américaines de 1935 à 1980 se sont déplacées de la gauche vers la droite et du bas vers le haut dans le premier quadrant. Elles n'ont pu passer au second quadrant comme le firent en leur temps la Corée du sud ou bien Taïwan. Bien au contraire, avec la reprimarisation, elles se sont déplacées de la droite vers la gauche au sein du premier quadrant. Faut-il alors lier la croissance modeste, relativement volatile et peu durable des principales économies latino-américaines à leur incapacité à augmenter le poids de leur manufacture dans le PIB? Peut-on expliquer la désindustrialisation du 
Brésil par leur reprimarisation et donc par leurs relations commerciales de plus en plus privilégiées avec la Chine ? Enfin peut-on alors attribuer à la Chine la responsabilité de cette faible croissance et aujourd'hui de la crise, crise qui serait provoquée par la baisse du cours des matières premières?

Le Brésil, ainsi que de nombreux autres pays d'Amérique latine ont échappé à la contrainte externe grâce à l'entrée de capitaux de l'étranger et à la hausse du cours des matières premières et de la forte augmentation des volumes exportés. Cette double hausse a été alimentée par la forte croissance exceptionnellement longue de la Chine. L'abondance de devises a déclenché un processus d'appréciation de la monnaie nationale de 2004 à 2011, sans que les gouvernements successifs aient cherché à le ralentir, sauf de manière erratique de 2011 à 2014, lors de la première présidence de Dilma Roussef.

Taux de change réel effectif du Brésil de février 1999 à juillet 2014, veille de la crise (base 100 en 2000)

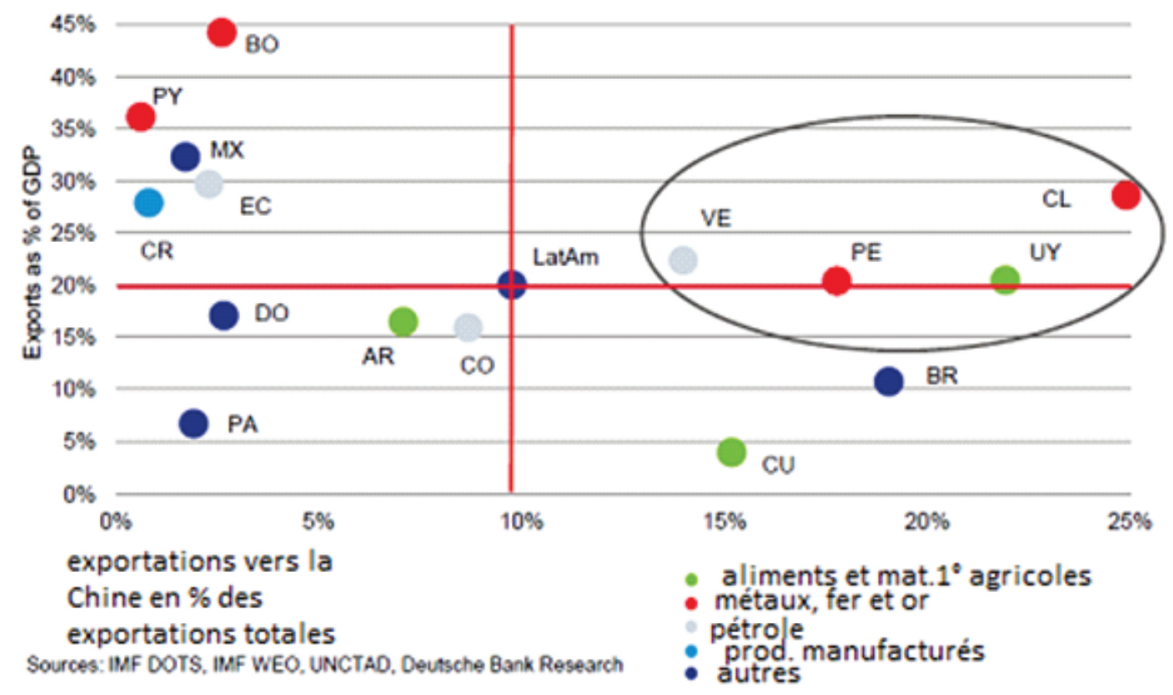

Repris de Nassif et alii (2015) ${ }^{5}$.

Afin de faciliter la lecture du graphique, rappelons qu'une courbe décroissante signifie une appréciation de la monnaie nationale. Les taux de change sont exprimés en termes réels afin de tenir compte des différentiels d'inflation avec les Etats-Unis

\footnotetext{
5 Nassif A?, Feijo C., Araujo E. (2015) Structural change and economic development: is Brazil catching up or falling behind? Cambridge Journal of Economics 2015, 39, 1307-1332
} 
L'appréciation de la monnaie nationale par rapport au dollar est un obstacle à une plus grande compétitivité, non seulement parce qu'elle rend moins coûteux les produits importés et à l'inverse plus chers les produits exportés, mais aussi parce qu'elle décourage l'investissement dans le secteur exposé, permet une spéculation immobilière et d'une manière générale réoriente les investissements dans les secteurs non exposés et dans le secteur produisant des matières premières. Au final le taux d'investissement peine à croitre, fléchit dans le secteur industriel et rend difficile le maintien d'un taux de croissance du PIB à un niveau élevé.

Taux d'investissement et d'épargne brute, taux de croissance du PIB, 2010-2015

\begin{tabular}{l|l|l|l|l|l|l}
\hline Taux & 2010 & 2011 & 2012 & 2013 & 2014 & 2015 \\
\hline Taux d'investissement & 20.5 & 20.6 & 20.7 & 20.9 & 20.2 & 18.2 \\
\hline Taux d'épargne & 17.9 & 18.5 & 18 & 18.4 & 16.2 & 14.4 \\
\hline Taux de croissance du PIB & 7.5 & 3.9 & 1.9 & 3 & 0.1 & -3.8 \\
\hline
\end{tabular}

Source: IBGE

La relative faiblesse du taux d'investissement dans le secteur industriel n'est pas propice à une augmentation de la productivité du travail et ce d'autant plus que l'effort en termes de recherche et développement n'est pas conséquent $(1 \%$ du PIB) surtout si on le compare a celui fait par la Corée du sud (plus de 4\%). La productivité du travail dans le secteur industriel croit en effet relativement peu : 17\% entre 2004 et 2015. Le salaire réel moyen augmente de 34\% dans la même période, davantage que la productivité.

Productivité et salaires réels dans l'industrie de transformation en monnaie nationale, base $100=2004$

\begin{tabular}{|c|c|c|c|c|c|c|c|c|c|c|c|c|}
\hline & 2004 & 2005 & 2006 & 2007 & 2008 & 2009 & 2010 & 2011 & 2012 & 2013 & 2014 & $2015^{*}$ \\
\hline 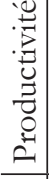 & 100 & 102 & 103.5 & 107.5 & 107.5 & 105.5 & 115 & 115 & 112.5 & 117 & 117 & 116 \\
\hline 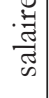 & 100 & 102.5 & 102.5 & 106 & 110 & 115 & 118.5 & 122.5 & 128.5 & 132 & 134 & 133 \\
\hline
\end{tabular}

Source: CEMEC, note 08/2015, *janvier- aout 2015 
Pour une base100 en 2004, l'indice des prix des importations de biens de consommation non durables est de 150 en 2014, à la veille de la crise et de la forte dépréciation du real, celui des biens de consommation durables est à 113, des biens intermédiaires à 120 , des biens d'équipement à 100 à la même date. Toujours pour une base 100 en 2004, l'indice du coût unitaire du travail dans l'industrie de transformation dépasse largement ceux des biens importés pour se situer à 221 (source FUNCEX, BACEN, élaboration CEMEC ). Les trois composantes de coût unitaire du travail évoluant défavorablement (appréciation de la monnaie qui se reflète dans les prix importés, hausse des salaires qui dépasse une productivité en très faible croissance), la compétitivité du pays décline fortement ; Elle baisse d'autant plus fortement que les biens produits sont sophistiqués, à l'exception de quelques branches comme l'aéronautique. On comprend dès lors que la balance commerciale de produits de l'industrie de transformation soit devenue négative en 2008, puis de plus en plus négative jusque 2014 (plus de 25 milliards de dollars de déficit ${ }^{6}$ ). L'augmentation de la demande, suite à l'augmentation des salaires, n'a pas engendré une augmentation de la production industrielle mais une augmentation des importations.

La production industrielle stagne et s'effondre avec la crise. Pour un indice 100 en moyenne en 2002 (donnée désaisonnalisée), elle atteint un pic de 105,5 en juin 2013, puis 99 en novembre 2014, et 85 en janvier 2016 et 83 en février 2016, malgré le nouvel essor des exportations, l'industrie poursuit son déclin (carta IEDI, $n^{\circ} 726$ ). Si on ne tient pas compte de la crise de 2015-2016, en onze ans l'industrie de transformation n'a pas cru. Passé un certain stade de développement, il est habituel de constater une baisse relative de la part du secteur industriel dans le PIB au profit des services, sans que pour autant il y ait nécessairement désindustrialisation. Le terme de désindustrialisation est en général réservé à une baisse absolue de la valeur ajoutée de l'industrie et/ou à une réduction relative du poids de l'industrie nationale dans l'industrie mondiale en économie ouverte. En Amérique Latine, ce phénomène a tendance à intervenir beaucoup plus tôt que dans les pays avancés, d'où le recours au qualificatif "précoce » utilisé lorsque le revenu par tête au début du processus de désindustrialisation correspond à la

${ }^{6}$ Ce n'est qu'à partir du second semestre que ce déficit se réduit suite à la maxi dépréciation de la monnaie (d'octobre 2014 à octobre 2015, la dépréciation nominale est de 47,24\% selon la Banque centrale, soit $37 \%$ en terme réel), les exportations régressant moins que les importations. Ce n'est qu'en fin 2015 que les exportations augmentent, les mois passant, de plus en plus fortement. Le solde négatif de la balance commerciale de produits industriels disparait alors et le solde de la balance commerciale devient de plus en plus positif malgré la chute des cours des matières premières. Pour le seul mois de mars 2016, il atteint 4,43 milliards de dollars. 
moitié de celui des pays avancés au moment où il débute. La part de l'industrie de transformation brésilienne dans l'industrie de transformation mondiale (en valeur ajoutée) est de 1,8\% en 2005 puis en 2011 de 1,7\% après avoir été de 2,7\%en 1980, selon la banque de données 2013 de l'UNCTAD. Selon la même source, en Chine, cette part était de 9,9\%, en 2005 et de 16,9\% en 2011. Elle baisse donc relativement au Brésil alors qu'elle augmente fortement en Chine. Les exportations de produits manufacturés régressent en termes relatifs au Brésil, passant de 53\% de la valeur des exportations en 2005 à 35\% en 2012, au profit des exportations de matières premières agricoles et minières et ce n'est que depuis février 2016 qu'elle croit à nouveau suite à la forte dévaluation et à la chute du cours des matières premières comme nous l'avons vu.

La faute à qui? A la Chine? Ou bien aux gouvernements brésiliens qui se sont succédés. La désindustrialisation observée fait penser à la "maladie hollandaise". On sait que celle-ci n’est pas inéluctable. La voie pour sortir du piège rentier est étroite mais elle existe. Les gouvernements brésiliens ont préféré ne pas l'emprunter, persuadés que la hausse du cours des matières premières allait se poursuivre et qu'elle permettrait, sans faire des réformes structurelles - comme celle de la fiscalité - afin d'éviter des conflits importants, de mener une politique visant à réduire les inégalités salariales, de diminuer la pauvreté en laissant les 1\% les plus riches s'enrichir davantage. La reprimarisation a consolidé des comportements rentiers présents depuis longtemps, legs du passé.

Une reprise de la croissance via une ré-industrialisation est possible. La période faste du boom des matières premières parait révolue. Reste que cette activité continue à procurer beaucoup de devises au Brésil. Celles-ci pourraient servir de tremplin pour une insertion plus positive dans la division internationale du travail. Qu'on songe aux exportations de produits agricoles. Celles-ci pourraient incorporer davantage de valeur ajoutée et être en adéquation avec l'évolution des consommations en Chine comme le préconise l'IPEA. D’une manière plus générale, la ré-industrialisation nécessite que cinq conditions soient satisfaites: 1 / un accroissement important de la productivité du travail diminuant le coût unitaire du travail; 2/ la remise en question de la logique des économies rentières qui limite le taux d'investissement à une portion congrue grâce à une politique industrielle active de l'Etat visant à aider des secteurs de pointe - et non l'ensemble de l'industrie - et pouvant nécessiter des mesures protectionnistes ciblées et temporaires; 3 / une politique active ayant pour objectif de neutraliser l'appréciation de la monnaie; 4/ une réforme fiscale permettant de diminuer les inégalités de revenus et d'améliorer le pouvoir d'achat de la population pauvre, 
vulnérable et des couches moyennes basses (émergentes) et de financer des services publics de qualité (éducation, santé...); 5/ une politique facilitant l'accès au crédit des pauvres et des couches vulnérables. Qu'une de ces conditions ne soit pas satisfaite rend plus aléatoire le succès d'une politique de croissance tirée par l'essor du marché intérieur.

\section{BIBLIOGRAFIA}

CARDIM DE CARVALHO, F. The narrow path for BraziL, Levy Economics Institute for Bard College, Policy Note, Estados-Unidos, 2016, pp.1-10

COSTA PINTO, E. y GONÇALVES, R. Globalização e poder efetivo: transformações globais sob efeito da ascensão chinesa, Economia e Sociedade, Campinas, Unicamp. IE. v. 24, n. 2 (54), Brasil, 2015, pp 449-479.

DUSSEL, P. E. y ORTIZ VELÁSQUEZ, S., Monitor de la OFDI de China en México, Año 1 - Número 1, 2016

DUSSEL P. E. A relação de comércio e de investimentos de América Latina com a China (2000-2012), uma nova forma de dependência, Revista Tempo du Mundo, Brasil, IPEA, vol 2 n 1, 2016, pp 123 -142,

FOSTER, M. China rebalancing:Blessing and curse for Latin America Research Briefing, Emerging Markets, Deutsche Bank Research, 2015, pp.1-6

HIRATUKA C. y SARTI F. Relações econômicas entre Brasil e China: análise dos fluxos de comércio e investimento direto estrangeiro, Revista Tempo du Mundo, Brasil, IPEA, vol 2 n 1, 2016, 2016, pp.83-98

MACEDO CINTRA, M. A. y COSTA PINTO, E. América latina y China en el siglo XXI: complementariedades y rivalidades, Revista Vozes en el Fenix, Brasil, 2013, pp 86-95,

SALAMA, P. China-Brasil: industrialización y desindustrialización temprana, Cuadernos de economia, 31(56), Colombia, 2012, pp. 223-252

SALAMA, P. Globalización comercial: desindustrialización prematura en América Latina e industrialización en Asia, Comercio Exterior, Vol. 62, Núm. 6, Mexico, 2012, pp. 34-44. 
SALAMA, P. Una globalización comercial acompañada de una nueva distribución cartográfica, Economía Institucional, Vol 14, n²7, Colombia, 2012, pp: 57-80. SALAMA, P. Les économies émergentes latino-américaines, entre cigales et fourmis, Armand Colin, Collection U, Paris, 2012.

SALAMA, P. China - Brasil, una comparacion instructiva, Foro Internacional, vol LV, n 220, Mexico, 2015, pp 515-624.

TREBAT, N. y AGUIAR DE MEDEIROS, C. Military Modernization in Chinese Technical Progress and Industrial Innovation, Review of Political economy, Inglaterra, Vol 26, Issue 2, 2014, pp 303-324

VIEIRA PEDRO, A., y BUAINAIN, A. M. y COVOLAN FIGUEIREDO, E. V. O Brasil alimentará a China ou a China engolirá o Brasil? Revista Tempo du Mundo, Brasil, IPEA, vol 2 n 1, 2016, pp.51-82

\section{Instituciones internationales}

OCDE: www.oecd.org

CEPAL: www.eclac.org

BID: www.iadb.org

ADB: www.adb.org 
